\title{
Quasi-electrostatic twisted waves in Lorentzian dusty plasmas
}

\author{
Kashif Arshad ${ }^{a, b, *}$, M. Lazar ${ }^{a, c}$, S. Poedts ${ }^{a}$ \\ ${ }^{a}$ Centre for Plasma Astrophysics (CPA), Celestijnenlaan 200B, 3001, Leuven, Belgium \\ ${ }^{\mathrm{b}}$ Pakistan Institute of Engineering and Applied Sciences, Islamabad, 45650, Pakistan \\ ${ }^{\mathrm{c}}$ Theoretical Physics IV, Ruhr-University Bochum, D-44780 Bochum, Germany
}

\section{A R T I C L E I N F O}

\section{Keywords:}

Orbital angular momentum

Laguerre Gaussian

Lorentzian

Helical

Kinetic theory

\begin{abstract}
A B S T R A C T
The quasi electrostatic modes are investigated in non thermal dusty plasma using non-gyrotropic Kappa distribution in the presence of helical electric field. The Laguerre Gaussian (LG) mode function is employed to decompose the perturbed distribution function and helical electric field. The modified dielectric function is obtained for the dust ion acoustic (DIA) and dust acoustic (DA) twisted modes from the solution of Vlasov-Poisson equation. The threshold conditions for the growing modes is also illustrated.
\end{abstract}

\section{Introduction}

Charged dust particles and dusty plasmas are ubiquitous in cosmic and astrophysical environments (Vladimirov et al., 2005) like interstellar-media, molecular dusty clouds, star forming dust clouds, Eagle nebula, and supernova remnants, our solar system (Mann et al., 2011) such as planetary rings systems and interplanetary media due to the presence of cometary dust particles, Jupiter's dusty rings (Hamilton and Kruger, 2008), cometary nuclei (Patzold et al., 2008) and Earth's mesosphere (Knappmiller et al., 2011). Most of such studies are based on the unrealistic (planar) electric field. Shukla, 2011, predicted dusty plasma modes by considering the non-planar (helical) electric field carrying orbital angular momentum (OAM).

The lasers carrying orbital angular momentum (Allen and Padgett, 2007; Grier, 2003) boosted a major scientific advancement development in various regimes like microscopy (Jesacher et al., 2005) and imaging (Jack et al., 2009), atomic and nano-particle manipulation (Padgett and Bowman, 2011), ultra-fast optical communications (Wang et al., 2012), quantum computing (Molina-Terriza et al., 2007), ionospheric radar facility to observe 3D plasma dynamics in ionosphere (Zolesi and Cander, 2014), photonic crystal fibre (Zhang et al., 2016), OAM entanglement of two photons (Oemrawsingh et al., 2005), twisted gravitational waves (Bialynicki-Birula and Bialynicka-Birula, 2016), ultra intense twisted laser pulses (Vieira et al., 2016) and astrophysics (Tamburini et al., 2011).

The interests for understanding the wave propagation in plasmas carrying orbital angular momentum have increased considerably in the last decade. Verbeeck and Schattschneider, 2010 studied the production and application of electron vortex beams. The magnetic tornadoes (Wedemeyer et al., 2013) and Alfvenic tornadoes (Shukla, 2013) exhibit spiral, ring and split morphologies and these have solution like Laguerre-Gaussian vortex beam. The magnetic tornadoes are supposed to be a rotating magnetic field structures in the solar atmosphere or chromospheric swirls while the Alfvenic tornadoes are the three dimensional modified kinetic Alfven wave. Leyser et al., 2009 experimentally created and pumped radio beam with helical wave front carrying OAM into the ionospheric plasma under High frequency Active Auroral Research Program (HAARP). Shukla et al., 2012 studied the stimulated scattering instabilities in dense quantum plasma for the coherent circularly polarized electromagnetic (CPEM) waves having orbital angular momentum. Mendonca et al., 2009 illustrated that some certain scattering phenomenon like stimulated Raman and Brillouin backscattering are responsible for the interaction between electrostatic and electromagnetic waves through orbital angular momentum. Shukla, 2012 obtained the dispersive shear Alfven wave (DSAW) modes from generalized ion vorticity and electron momentum equations aligned with the magnetic field in a magnetized plasma, in Earth's auroral zone, solar atmosphere (Khotyaintsev, 2006; Khotyaintsev et al., 2006), and in laboratory plasma e.g., Large Plasma Device (LAPD) at University of California, Los Angeles (Popovich et al., 2010).

Barnett et al., 2016 motivated the study of some new features of orbital angular momentum (OAM) in plasmas e.g. the generation of waves carrying OAM with dielectric resonator antenna array (Akram et al., 2016), bump on tail twisted instability in cold atomic clouds

\footnotetext{
* Corresponding author. Centre for Plasma Astrophysics (CPA), Celestijnenlaan 200B, 3001, Leuven, Belgium.

E-mail address: kashif.arshad.butt@gmail.com (K. Arshad).
} 
(Rodrigues et al., 2016) and damping of the twisted waves in Maxwellian distributed multi-component dusty plasma (Ali et al., 2016).

In laboratory experiments and most of the astrophysical environments (Arshad and Mahmood, 2010; 2011) the charged particles exhibit non-Maxwellian or non-thermal distribution. These non-Maxwellian distributions are well reproduced by the power-laws or the so-called Kappa distribution functions (Pierrard and Lazar, 2010; Lazar et al., 2012), which recently were applied for modeling the twisted plasma waves, such as Langmuir (electron) twisted waves and ion acoustic twisted waves (Arshad et al., 2016). In space special form of plasma like permeating plasmas (Arshad et al., 2014a,b,c), streaming plasmas (Novo et al., 2015; Lazar et al., 2008; Youdin et al., 2005; Bret, 2009; Khalil, 2015; Torney et al., 2006) and drifting plasmas (Davidson, 2014) exhibit various novel instabilities in the presence of neutral and charged dust particles. In the last decades, numerous linear and non-linear studies have been proposed to investigate ion acoustic and acoustic waves in dusty plasmas (Barkan et al., 1996; Pieper and Goree, 1996; Mamun et al., 1996; Frank Verheest, 1992) for example experimental study of dust ion acoustic waves, dispersion relation of dust acoustic waves and non-linear dust acoustic waves. Recently, some new features were included in the study of dust acoustic and dust ion acoustic waves like ion acoustic waves in cosmic dusty plasmas (Gao and Tian, 2007; 2006), spherical dust ion acoustic waves (Jukui and He, 2003; Tian and Gao, 2005) and Bohm criterion in dusty plasmas, dusty clusters and non-degenerate quantum dusty plasma (Fouial et al., 2016; Bessaa et al., 2016). Also notice that Ali et al., 2016 investigated twisted ion acoustic and acoustic waves for dusty plasmas conditions, but restricting to standard Maxwellian distribution to describe the plasma components, and without any drift of the electrons.

In this manuscript kinetic theory is applied to study the dust ion acoustic and dust acoustic waves in the presence of electric field carrying orbital angular momentum in a plasma containing non-Maxwellian drifted electrons, ions and dust particles described by the generalized Lorentzian or Kappa distribution functions. The superthermal Kappa distributed electrons acquire drift velocity along the longitudinal direction. This drift velocity of electrons act as the main source of energy, which support the growing instabilities of quasi-electrostatic waves in Lorentzian dusty plasmas. We though consider the distinct Kappa indices for the electrons, ions and dust particles i.e., $\kappa_{e}, \kappa_{i}$ and $\kappa_{d}$ respectively, which may have some contribution to accomplish the growing modes in quasi-electrostatic dusty plasmas but the role of Lorentzian distributed drifted superthermal electrons is dominant.

\section{Plasma dispersion function with helical electric field}

The plasma dielectric function $\varepsilon\left(\omega, k, l q_{\theta}\right)$ for the quasi-electrostatic waves carrying orbital angular momentum of the electric field in a dusty plasma can be written as (Arshad et al., 2015; 2016),

$\varepsilon\left(\omega, k, l q_{\theta}\right)=1+\sum_{\alpha=e, i, d} \frac{\omega_{p \alpha}^{2}}{k^{2}} \int \frac{\mathbf{q}_{e f f} \cdot \partial_{\mathbf{v}} f_{0 \alpha}}{\left(\omega-\mathbf{q}_{e f f} \cdot \mathbf{v}\right)} d \mathbf{v}$,

where $\omega_{p \alpha}=\sqrt{4 \pi n_{0 \alpha} q_{\alpha}^{2} / m_{\alpha}}$ is the plasma frequency of the $\alpha$-species and $q_{\alpha}=e, Z_{i} e$ and $Z_{d} e$ for the electrons, ions and dust respectively. The term $\mathbf{q}_{\text {eff }}$ is total wave number in the presence of helical electric field.

$\mathbf{q}_{e f f}=-i q_{r} \widehat{\mathbf{e}}_{r}+l q_{\theta} \widehat{\mathbf{e}}_{\theta}+\left(k-i q_{z}\right) \widehat{\mathbf{e}}_{z}$.

The parameters $q_{j}(j=r, z)$ and $q_{\theta}$ can be defined in term of $F_{p l}$ : Laguerre Gaussian (LG) mode function as;

$q_{j}=\int_{0}^{\infty} F_{p l} \partial_{j} F_{p l} r d r, q_{\theta}=\int_{0}^{\infty} F_{p l}^{2} d r$.

Here $F_{p l}$ is Laguerre Gaussian (LG) mode function, which can be expressed as;
$F_{p l}(r, z)=C_{p l} X^{|l|} L_{p}^{|l|}(X) \exp (-X / 2)$,

where $X=r^{2} / w^{2}(z)$ such that $w(z)$ is the beam waist. Here integers $p$ and $l$ are the radial and angular mode numbers, while $\theta$ denotes the azimuthal angle. The normalization constants $C_{p l}$ and the associated Laguerre polynomials $L_{p}^{|l|}(X)$ have the following expressions

$C_{p l}=\sqrt{(l+p) ! / 4 \pi p !}$,

and

$L_{p}^{|l|}(X)=\exp (X) d^{p} / d X^{p}\left[X^{l+p} \exp (-X)\right] / p ! X$.

The plasmas susceptibility $\chi\left(\omega, k, l q_{\theta}\right)$ of quasi-electrostatic twisted waves in dusty plasma can be written as

$\chi\left(\omega, k, l q_{\theta}\right)=\sum_{\alpha=e, i, d} \frac{\omega_{p \alpha}^{2}}{k^{2}} \int \frac{\mathbf{q}_{e f f} \cdot \partial_{\mathbf{v}} f_{0 \alpha}}{\left(\omega-\mathbf{q}_{e f f} \cdot \mathbf{v}\right)} d \mathbf{v}$.

The susceptibility of twisted quasi-electrostatic waves in unmagnetized dusty plasma carrying orbital angular momentum can be simplified as,

$\chi\left(\omega, l q_{\theta}, k\right)=\sum_{\alpha=e, i, d} \frac{\omega_{p \alpha}^{2}}{k^{2}} \int \frac{\mathbf{q}_{e f f} \cdot \partial_{\mathbf{v}} f_{0 \alpha} d \mathbf{v}}{\left(\omega-k v_{z}-l q_{\theta} v_{\theta}\right)}$.

The modified resonance condition $\omega=k v_{z}+l q_{\theta} v_{\theta}$ resembles the Landau cyclotron resonance for the magneto plasma case (Alexandrov et al., 1984). By substituting the value of non-gyrotropic Kappa distribution in above equation, we obtain the following form of modified plasma dispersion function in the presence of helical electric field (Arshad et al., 2017);

$Z_{\kappa, l q_{\theta}}\left(\xi_{z}, \xi_{\theta}\right)=\frac{2}{\theta^{2}}\left[\frac{2(\kappa-1)}{\kappa}+\xi_{z} Z^{*}\left(\xi_{z}\right)+\xi_{\theta} Z^{*}\left(\xi_{\theta}\right)\right]$

where $Z^{*}\left(\xi_{z}\right)$ and $Z^{*}\left(\xi_{\theta}\right)$ are the longitudinal and azimuthal components of plasma dispersion function, which has the expression;

$$
\begin{aligned}
Z^{*}\left(\xi_{z}\right)= & \frac{1}{\sqrt{\pi}} \frac{\Gamma(\kappa+1)}{\kappa^{3 / 2} \Gamma(\kappa-1 / 2)} \\
& \times \int_{-\infty}^{\infty} \frac{d \varsigma_{z}}{\left(\varsigma_{z}-\xi_{z}\right)}\left[1+\frac{\varsigma_{z}^{2}}{\kappa}\right]^{-\kappa-1}
\end{aligned}
$$

and

$$
\begin{aligned}
Z^{*}\left(\xi_{\theta}\right)= & \frac{1}{\sqrt{\pi}} \frac{\Gamma(\kappa+1)}{\kappa^{3 / 2} \Gamma(\kappa-1 / 2)} \\
& \times \int_{-\infty}^{\infty} \frac{d \varsigma_{\theta}}{\left(\varsigma_{\theta}-\xi_{\theta}\right)}\left[1+\frac{\varsigma_{\theta}^{2}}{\kappa}\right]^{-\kappa-1}
\end{aligned}
$$

while the terms $\xi_{z}$ and $\xi_{\theta}$ are defined as

$\xi_{z}=\frac{\left(u_{z}-v_{d}\right)}{\theta} ; \xi_{\theta}=\frac{\left(u_{\theta}-v_{d}\right)}{\theta}$,

The non-gyrotropic drifted Kappa distribution (Arshad et al., 2017) is define as;

$$
\begin{aligned}
f_{0 \alpha}= & \sum_{\alpha=e, i, d} \frac{n_{\alpha 0}}{\pi^{3 / 2} \theta_{\alpha}^{3}} \frac{\Gamma\left(\kappa_{\alpha}+1\right)}{\kappa_{\alpha}^{3 / 2} \Gamma\left(\kappa_{\alpha}-1 / 2\right)} \\
& {\left[1+\frac{1}{\kappa_{\alpha} \theta_{\alpha}^{2}}\left[\left(v_{z}-v_{0 \alpha}\right)^{2}+v_{r}^{2}+v_{\theta}^{2}\right]\right]^{-\kappa_{\alpha}-1}, }
\end{aligned}
$$

where $\kappa_{\alpha}$ is the spectral index of the $\alpha$-species and $\theta_{\alpha}=\left(2 \kappa_{\alpha}-3 / \kappa_{\alpha}\right)^{1 / 2} v_{T_{\alpha}}$ 
with $\kappa>3 / 2$ is the Lorentzian thermal velocity for non-thermal species such that $v_{T_{\alpha}}=\left(T_{\alpha} / m_{\alpha}\right)^{1 / 2}$ is the thermal velocity and $v_{0 \alpha}$ is the drift velocity along the $\mathrm{z}$-direction. In our studies the superthermal electrons have drift velocity while the for the case of ions and dust the drift velocity is consider to be very small that it approaches to zero.

\section{Dust ion acoustic (DIA) and dust acoustic (DA) twisted modes}

The dielectric functions of the quasi-electrostatic twisted (helical) modes in non-Maxwellian (Kappa distributed) dusty plasmas i.e., dust ion acoustic (DIA) and dust acoustic (DA) twisted (helical) modes can be written as (Arshad et al., 2017),

$$
\begin{aligned}
\varepsilon\left(\omega, k, l q_{\theta}\right)= & 1+\frac{2 \omega_{p e}^{2}}{k^{2} \theta_{e}^{2}}\left[\frac{2 \kappa_{e}-1}{\kappa_{e}}+\xi_{z_{e}} Z\left(\xi_{z_{e}}\right)\right. \\
& \left.+\xi_{\theta_{e}} Z\left(\xi_{\theta_{e}}\right)\right]+\frac{2 \omega_{p_{i}}^{2}}{k^{2} \theta_{i}^{2}} \\
& \times\left[\frac{2 \kappa_{i}-1}{\kappa_{i}}+\xi_{z_{i}} Z\left(\xi_{z_{i}}\right)+\xi_{\theta_{i}} Z\left(\xi_{\theta_{i}}\right)\right] .
\end{aligned}
$$

and

$$
\begin{aligned}
\varepsilon\left(\omega, k, l q_{\theta}\right)=1 & +\frac{2 \omega_{p e}^{2}}{k^{2} \theta_{e}^{2}}\left[\frac{2 \kappa_{e}-1}{\kappa_{e}}+\xi_{z_{e}} Z\left(\xi_{z_{e}}\right)\right. \\
& \left.+\xi_{\theta_{e}} Z\left(\xi_{\theta_{e}}\right)\right]+\frac{2 \omega_{p i}^{2}}{k^{2} \theta_{i}^{2}}\left[\frac{2 \kappa_{i}-1}{\kappa_{i}}\right. \\
& \left.+\xi_{z_{i}} Z\left(\xi_{z_{i}}\right)+\xi_{\theta_{i}} Z\left(\xi_{\theta_{i}}\right)\right] \\
& +\frac{2 \omega_{p d}^{2}}{k^{2} \theta_{d}^{2}}\left[\frac{2 \kappa_{d}-1}{\kappa_{d}}+\xi_{z_{d}} Z\left(\xi_{z_{d}}\right)+\xi_{\theta_{d}} Z\left(\xi_{\theta_{d}}\right)\right] .
\end{aligned}
$$

here $Z\left(\xi_{z_{e}}\right)\left[Z\left(\xi_{\theta_{e}}\right)\right], Z\left(\xi_{z_{i}}\right)\left[Z\left(\xi_{\theta_{i}}\right)\right]$ and $Z\left(\xi_{z_{d}}\right)\left[Z\left(\xi_{\theta_{d}}\right)\right]$ are the planar [azimuthal] plasma dispersion functions of electrons, ions and dust particles and the terms $\left(\xi_{z_{e}}, \xi_{\theta_{e}}, \xi_{z_{i}}, \xi_{\theta_{i}}, \xi_{z_{d}}\right.$ and $\left.\xi_{\theta_{d}}\right)$ have following expressions;

$\xi_{z e}=\frac{1}{\theta_{e}}\left(\frac{\omega}{k}-v_{0 e}\right) ; \quad \xi_{\theta_{e}}=\frac{1}{\theta_{e}} \frac{\omega}{l q_{\theta}}$,

$\xi_{z_{i}}=\frac{1}{\theta_{i}}\left(\frac{\omega}{k}\right) ; \quad \xi_{\theta_{i}}=\frac{1}{\theta_{i}}\left(\frac{\omega}{l q_{\theta}}\right)$,

$\xi_{z_{d}}=\frac{1}{\theta_{d}}\left(\frac{\omega}{k}\right) ; \quad \xi_{\theta_{d}}=\frac{1}{\theta_{d}}\left(\frac{\omega}{l q_{\theta}}\right)$.

By expansion of plasma dispersion function under appropriate limit and equating the real part of the dielectric functions to zero, we can calculate the dispersion spectrum of the quasi-electrostatic twisted modes in dusty plasmas (i.e., DIA and DA twisted modes) as given in Eq.[17] and Eq.[18];

$\omega_{r}^{2}=\frac{k^{2} \omega_{p_{i}}^{2} \lambda_{D e}^{2}}{\Upsilon^{2}}\left[\left(\frac{\kappa_{e}-3 / 2}{\kappa_{e}-1 / 2}\right) \frac{1+\Upsilon^{2}}{1+k^{2} \lambda_{k e}^{2}}\right]$.

and

$\omega_{r}^{2}=\frac{k^{2} \omega_{p_{d}}^{2} \lambda_{\kappa d}^{2}}{\left[1+k^{2} \lambda_{\kappa d}^{2}\right]} \frac{\left(1+\Upsilon^{2}\right)}{\Upsilon^{2}}$

Here $r=k / l q_{\theta}$ is the ratio of the conventional $(k)$ and azimuthal wave number $\left(l q_{\theta}\right)$ and $\left(\lambda_{k e}\right.$ and $\left.\lambda_{k d}\right)$ are the modified Debye (screening) length for the DIA and DA twisted (helical) modes such that; $\lambda_{\kappa e}=\left(\frac{\kappa_{e}-3 / 2}{\kappa_{e}-1 / 2}\right)^{1 / 2} \lambda_{D e}$

$\lambda_{\kappa d}^{-2}=\left(\frac{2 \kappa_{e}-1}{2 \kappa_{e}-3}\right) \lambda_{D e}^{-2}+\left(\frac{2 \kappa_{i}-1}{2 \kappa_{i}-3}\right) \lambda_{D i}^{-2}$.

The growth rate $(\gamma)$ can be obtained by substituting the values of real $\operatorname{Re}\left[\varepsilon\left(\omega, k, l q_{\theta}\right)\right]$ and imaginary $\operatorname{Im}\left[\varepsilon\left(\omega, k, l q_{\theta}\right)\right]$ parts of the dielectric function in the following expression;

$\gamma=-\left(\frac{\partial \operatorname{Re}\left[\varepsilon\left(\omega, k, l q_{\theta}\right)\right]}{\partial \omega_{r}}\right)^{-1} \operatorname{Im}\left[\varepsilon\left(\omega, k, l q_{\theta}\right)\right]$.

The normalized growth rates $\gamma / \omega_{r}$ of quasi-electrostatic DIA and DA twisted modes are given in Eq.[21] and Eq.[26];

$$
\begin{aligned}
\frac{\gamma}{\omega_{r}}=\frac{\sqrt{\pi}\left(\kappa_{e}-3 / 2\right)^{3 / 2}}{\left(\kappa_{e}-1 / 2\right)^{3 / 2}\left(1+k^{2} \lambda_{\kappa e}^{2}\right)^{3 / 2}} \\
\times \frac{\left(1+\Upsilon^{2}\right)^{1 / 2}}{r\left(1-\eta Z_{d}\right)^{3 / 2}} \\
\times\left[\left(1-\eta Z_{d}\right) \sqrt{\frac{m_{e}}{m_{i}}} \gamma_{e}-\left(\frac{T_{e}}{T_{i}}\right)^{3 / 2} \gamma_{i}\right] .
\end{aligned}
$$

Here $\eta=n_{d 0} / n_{i 0}$ is ratio of background number densities of dust particles to ions and $Z_{d}$ is dust charging parameter. The more simplified form of $\gamma_{e}=\left(\gamma_{e z}+\gamma_{e \theta}\right)$ can be attained by inserting the value of $\omega_{r}$ in the expressions of $\gamma_{e z}$ and $\gamma_{e \theta}$. The normalized expressions of the planar $\gamma_{e z}$ and azimuthal $\gamma_{e \theta}$ components of electrons are given as;

$$
\begin{aligned}
\gamma_{e z}=\frac{\kappa_{e} !}{\left(\kappa_{e}-3 / 2\right)^{3 / 2} \Gamma\left(\kappa_{e}-1 / 2\right)} \\
\times\left[\frac{\left(\kappa_{e}-1 / 2\right)^{1 / 2}\left(1+k^{2} \lambda_{\kappa e}^{2}\right)^{1 / 2}}{\left(\kappa_{e}-3 / 2\right)^{1 / 2}}\right. \\
\left.\times \frac{\left.r \sqrt{1-\eta Z_{d}} \frac{v_{0 e}}{\left(1+r^{2}\right)^{1 / 2}}-1\right]}{C_{s}}\right]
\end{aligned}
$$

and

$$
\begin{aligned}
\gamma_{e \theta}=\frac{\kappa_{e} ! \Upsilon}{\left(\kappa_{e}-3 / 2\right)^{3 / 2} \Gamma\left(\kappa_{e}-1 / 2\right)} \\
\times\left(\begin{array}{l}
1+\Upsilon^{2} \frac{\left(\kappa_{e}-3 / 2\right)}{\left(2 \kappa_{e}-3\right)\left(\kappa_{e}-1 / 2\right)} \frac{m_{e}}{m_{i}} \\
\times \frac{1+\Upsilon^{2}}{\left(1+k^{2} \lambda_{\kappa e}^{2}\right)} \frac{1}{\left(1-\eta Z_{d}\right)}
\end{array}\right)^{-\kappa_{e}-1}
\end{aligned}
$$

The growth term of ions $\gamma_{i}^{\prime}=\left(\gamma_{i z}{ }^{\prime}+\gamma_{i \theta^{\prime}}\right)$ is also modified by using the value of $\omega_{r}$ in the expressions of $\gamma_{i z}$ and $\gamma_{i \theta}$, as follows;

$$
\begin{aligned}
\gamma_{i z}=\frac{\kappa_{i} !}{\left(2 \kappa_{i}-3\right)^{3 / 2} \Gamma\left(\kappa_{i}-1 / 2\right)} \\
\times\left(\begin{array}{c}
1+\frac{\left(\kappa_{e}-3 / 2\right)}{\left(2 \kappa_{i}-3\right)\left(\kappa_{e}-1 / 2\right)} \frac{T_{e}}{T_{i}} \\
\times \frac{1+\Upsilon^{2}}{r^{2}\left(1+k^{2} \lambda_{\kappa e}^{2}\right)} \frac{1}{\left(1-\eta Z_{d}\right)}
\end{array}\right)^{-\kappa_{i}-1}
\end{aligned}
$$

and 


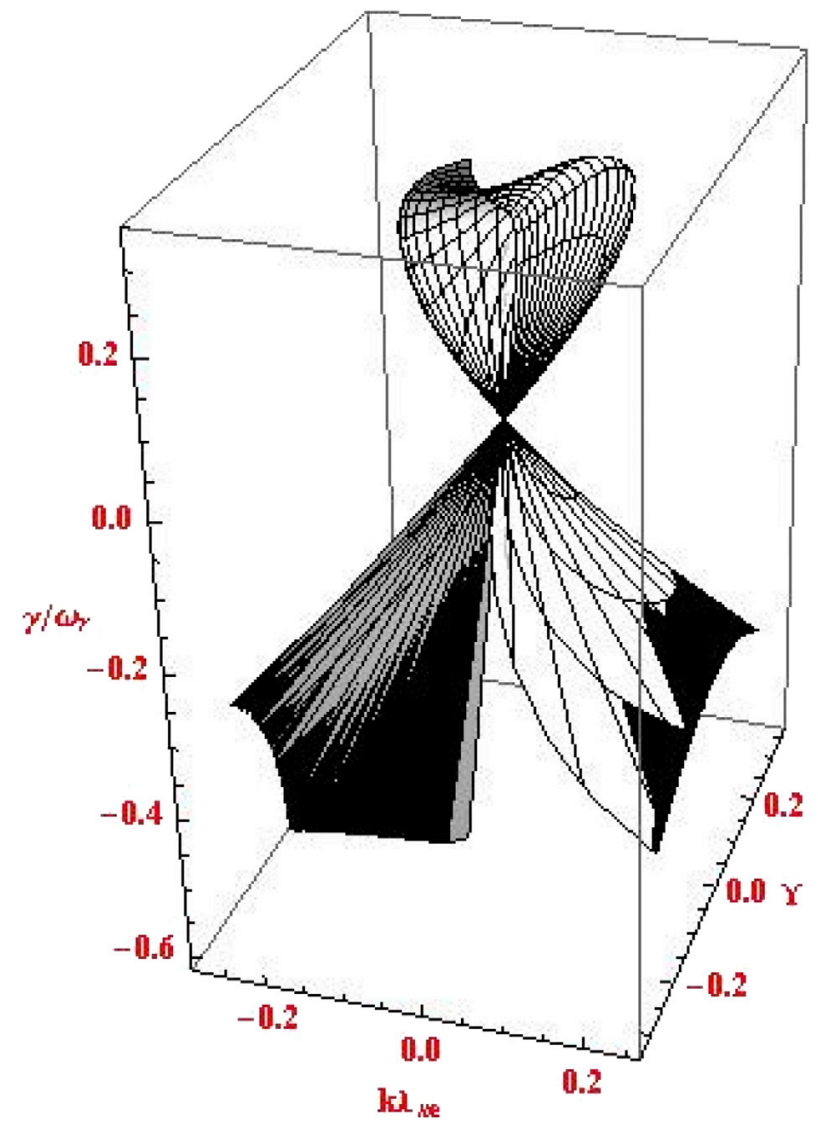

Fig. 1. The plots of normalized growth rate $\gamma / \omega_{r}$ of quasi-electrostatic DIA twisted modes are presented against the normalized wave number $k \lambda_{k e}$ and azimuthal parameter $\Upsilon=$ $k / l q \theta$ with $\eta Z_{d}=0.01, v_{0 e} / C_{s}=2.5$ and $T_{e} / T_{i}=30$.

$$
\begin{aligned}
\gamma_{i \theta}=\frac{\kappa_{i} ! \Upsilon}{\left(2 \kappa_{i}-3\right)^{3 / 2} \Gamma\left(\kappa_{i}-1 / 2\right)} \\
\times\left(\begin{array}{l}
1+\frac{\left(\kappa_{e}-3 / 2\right)}{\left(2 \kappa_{i}-3\right)\left(\kappa_{e}-1 / 2\right)} \frac{T_{e}}{T_{i}} \\
\times \frac{1+\Upsilon^{2}}{\left(1+k^{2} \lambda_{\kappa e}^{2}\right)} \frac{1}{\left(1-\eta Z_{d}\right)}
\end{array}\right)
\end{aligned}
$$

After applying these operations, the normalized growth rate $\gamma / \omega_{r}$ is written as;

$$
\begin{aligned}
\frac{\gamma}{\omega_{r}}=\frac{\sqrt{\pi}}{2} & \frac{1}{\left[1+k^{2} \lambda_{\kappa d}^{2}\right]^{3 / 2}} \\
\times & \Upsilon \frac{\left(1+\Upsilon^{2}\right)^{1 / 2}}{\Upsilon\left[\left(\frac{2 \kappa_{e}-1}{2 \kappa_{e}-3}\right) \frac{T_{i}}{T_{e}}\left(1-\eta Z_{d}\right)+\left(\frac{2 \kappa_{i}-1}{2 \kappa_{i}-3}\right)\right]^{3 / 2}} \\
\times & {\left[\begin{array}{l}
\left(1-\eta Z_{d}\right) \sqrt{\eta Z_{d}^{2} \frac{m_{e}}{m_{d}}}\left(\frac{T_{i}}{T_{e}}\right)^{3 / 2} \gamma_{e} \\
\left.-\left(\eta Z_{d}^{2} \frac{m_{i}}{m_{d}}\right)^{1 / 2} \gamma_{i}-\left(\eta Z_{d}^{2} \frac{T_{i}}{T_{d}}\right)^{3 / 2} \gamma_{d}\right] .
\end{array}\right.}
\end{aligned}
$$

To simplify the normalized growth rate $\gamma / \omega_{r}$ of the DATM, we have to simplify the growth rates of all the plasma components. The normalized growth rate of electron's contribution $\gamma_{e}=\left[\gamma_{e z}+\gamma_{e \theta}\right]$ is shown as;

$$
\begin{aligned}
\gamma_{e z}=\frac{\kappa_{e} !}{\left(2 \kappa_{e}-3\right)^{3 / 2} \Gamma\left(\kappa_{e}-1 / 2\right)} \\
\times\left[\left\{\left(\frac{2 \kappa_{e}-1}{2 \kappa_{e}-3}\right) \frac{T_{i}}{T_{e}}\left(1-\eta Z_{d}\right)+\left(\frac{2 \kappa_{i}-1}{2 \kappa_{i}-3}\right)\right\}\right. \\
\left.\times \frac{\Upsilon\left(1+k^{2} \lambda_{\kappa d}^{2}\right)^{1 / 2}}{\left(1+r^{2}\right)^{1 / 2}} \frac{v_{d}}{\left(\eta Z_{d}^{2}\right)\left(T_{i} / m_{d}\right)}-1\right],
\end{aligned}
$$

and

$$
\begin{aligned}
\gamma_{e \theta}= & \frac{\kappa_{e} ! \Upsilon}{\left(2 \kappa_{e}-3\right)^{3 / 2} \Gamma\left(\kappa_{e}-1 / 2\right)} \\
\times & \left(\begin{array}{l}
1+\frac{1}{\left(2 \kappa_{e}-3\right)} \frac{\left(1+\Upsilon^{2}\right)}{\left[1+k^{2} \lambda_{\kappa d}^{2}\right]} \frac{T_{i}}{T_{e}} \\
\times \frac{\left(\eta Z_{d}^{2}\right)\left(m_{e} / m_{d}\right)}{\left[\left(\frac{2 \kappa_{e}-1}{2 \kappa_{e}-3}\right) \frac{T_{i}}{T_{e}}\left(1-\eta Z_{d}\right)+\left(\frac{2 \kappa_{i}-1}{2 \kappa_{i}-3}\right)\right]}
\end{array}\right)
\end{aligned}
$$

The normalized growth rate due to ions contribution $\gamma_{i}=\left[\gamma_{i z}+\gamma_{i \theta}\right]$ has following expressions;

$$
\begin{aligned}
& \gamma_{i z}=\frac{\Gamma\left(\kappa_{i}+1\right)}{\left(2 \kappa_{i}-3\right)^{3 / 2} \Gamma\left(\kappa_{i}-1 / 2\right)}, \\
& \text { and } \\
& \qquad \gamma_{i \theta}=\frac{\Gamma\left(\kappa_{i}+1\right) \Upsilon}{\left(2 \kappa_{i}-3\right)^{3 / 2} \Gamma\left(\kappa_{i}-1 / 2\right)} \\
& \qquad\left(\begin{array}{c}
1+\frac{1}{\left(2 \kappa_{i}-3\right)} \frac{\left(1+\Upsilon^{2}\right)}{\left[1+k^{2} \lambda_{\kappa d}^{2}\right]} \\
\times \frac{\left(\eta Z_{d}^{2}\right)\left(m_{i} / m_{d}\right)}{\left[\left(\frac{2 \kappa_{e}-1}{2 \kappa_{e}-3}\right) \frac{T_{i}}{T_{e}}\left(1-\eta Z_{d}\right)+\left(\frac{2 \kappa_{i}-1}{2 \kappa_{i}-3}\right)\right]}
\end{array}\right)^{-\kappa_{i}-1}
\end{aligned}
$$

and in case of dust particles $\gamma_{d}=\left[\gamma_{d z}+\gamma_{d \theta}\right]$ with

$$
\begin{aligned}
\gamma_{d z}=\frac{\Gamma\left(\kappa_{d}+1\right)}{\left(2 \kappa_{d}-3\right)^{3 / 2} \Gamma\left(\kappa_{d}-1 / 2\right)} \\
\times\left(\begin{array}{c}
1+\frac{1}{\left(2 \kappa_{d}-3\right)} \frac{\left(1+\Upsilon^{2}\right)}{\Upsilon^{2}\left[1+k^{2} \lambda_{\kappa d}^{2}\right]} \frac{T_{i}}{T_{d}} \\
\times \frac{\left(\eta Z_{d}^{2}\right)}{\left[\left(\frac{2 \kappa_{e}-1}{2 \kappa_{e}-3}\right) \frac{T_{i}}{T_{e}}\left(1-\eta Z_{d}\right)+\left(\frac{2 \kappa_{i}-1}{2 \kappa_{i}-3}\right)\right]}
\end{array}\right)^{-\kappa_{d}-1}
\end{aligned}
$$

and

$$
\begin{aligned}
& \gamma_{d \theta}= \frac{\Gamma\left(\kappa_{d}+1\right)}{\left(2 \kappa_{d}-3\right)^{3 / 2} \Gamma\left(\kappa_{d}-1 / 2\right)} \\
& \times\left(\begin{array}{c}
1+\frac{1}{\left(2 \kappa_{d}-3\right)} \frac{\left(1+\Upsilon^{2}\right)}{\Upsilon^{2}\left[1+k^{2} \lambda_{\kappa d}^{2}\right]} \frac{T_{i}}{T_{d}} \\
\times \frac{\left(\eta Z_{d}^{2}\right)}{\left[\left(\frac{2 \kappa_{e}-1}{2 \kappa_{e}-3}\right) \frac{T_{i}}{T_{e}}\left(1-\eta Z_{d}\right)+\left(\frac{2 \kappa_{i}-1}{2 \kappa_{i}-3}\right)\right]}
\end{array}\right)^{-\kappa_{d}-1}
\end{aligned}
$$

\section{Results and discussion}

In this section, the three dimensional plots of normalized growth rate $\gamma / \omega_{r}$ are shown for the quasi-electrostatic dust ion acoustic (DIA) and dust acoustic (DA) twisted modes. The effects of various parameters like 


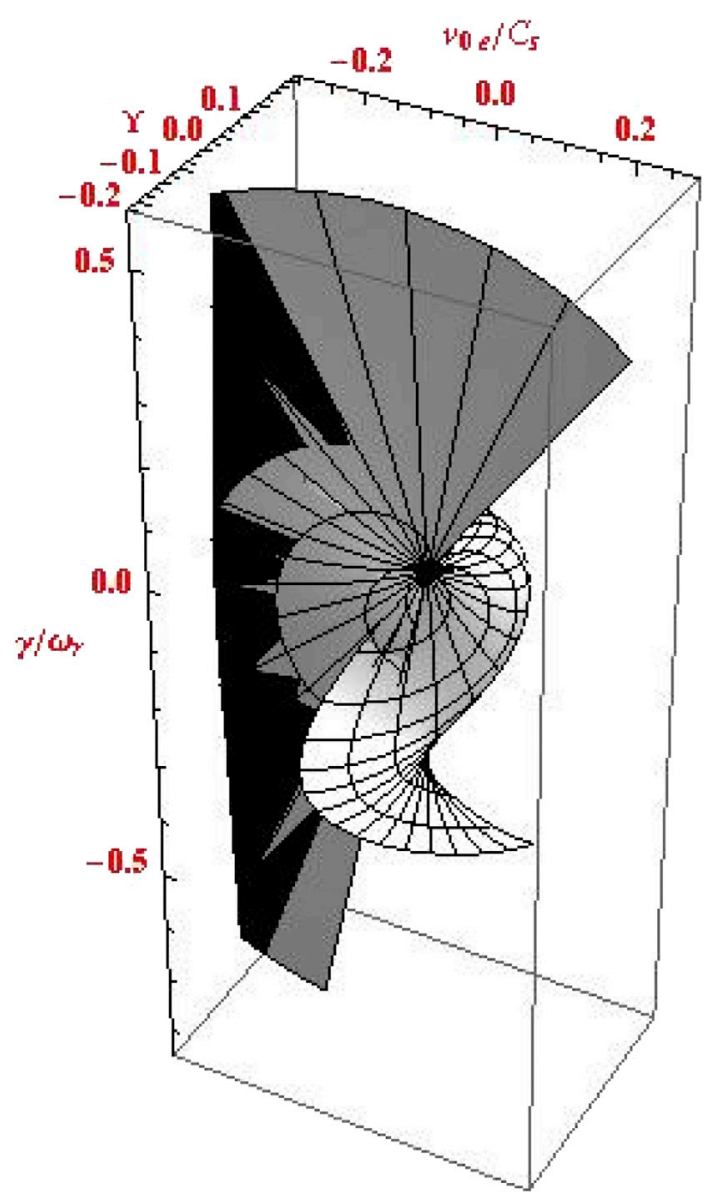

Fig. 2. The plots of normalized growth rate $\gamma / \omega_{r}$ of quasi-electrostatic DIA twisted modes are presented against the normalized drift velocity $v_{0 e} / C_{s}$ and azimuthal parameter $\Upsilon=$ $k / l q \theta$ with $\eta Z_{d}=0.01, k \lambda_{\kappa e}=0.1$ and $T_{e} / T_{i}=30$.

normalized wave numbers $k \lambda_{\kappa e}$ and $k \lambda_{\kappa d}$, normalized drift velocities $v_{0 e} / C_{s}$ and $v_{0 e} /\left(T_{i} / m_{d}\right)$, azimuthal parameter $\Upsilon=k / l q \theta$ and $\eta Z_{d}$ on the normalized growth rate $\gamma / \omega_{r}$ are illustrated graphically and physically of the quasi-electrostatic DIA and DA twisted modes.

Fig. 1 describes the spherical three dimensional plot of the growth rate $\gamma / \omega_{r}$ of the quais-electrostatic DIA twisted wave against the normalized wave number $k \lambda_{k e}$ and azimuthal parameter $\Upsilon=k / l q \theta$. It is evident from the figure that with increasing values $k \lambda_{k e}$ and $\Upsilon=k / l q \theta$, the growth rate of the wave increases. The physical interpretation of this phenomena is that the normalized wave number $k \lambda_{k e}$ belongs to presence of large number of probable superthermal drifted electrons that can transfer their energy to the wave. Because the larger value of $\Upsilon=k / l q \theta$ reveals that the value of longitudinal wave vector $k$ is if larger as compare to $l q \theta$, then wave can grow to larger magnitude in comparison to the smaller value of longitudinal wave vector $k$. The steepness of the curvature increases at lower $\Upsilon$ because of larger $l q \theta$ and decreases at higher $\Upsilon$ due to smaller $l q \theta$. It is depicted that the phasor wave number $l q \theta$ if dominates the spatial wave number $k$, then the wave is less probable to propagate in the longitudinal direction and consequently grows to smaller extent.

Fig. 2 presents the three dimensional spherical plots of the growth rate $\gamma / \omega_{r}$ of the quais-electrostatic DIA twisted wave against the normalized drift velocity $v_{0 e} / C_{s}$ and azimuthal parameter $\mathrm{r}=k / \mathrm{lq \theta}$. It is clear from the figure that by increasing the parameters $v_{0 e} / C_{s}$ and $\Upsilon=k / l q \theta$, the growth rate of the wave rate of the wave increases. The drift velocity $v_{0 e} / C_{s}$ contributes as an energy source for the growth rate of the wave. If the drift velocity $v_{0 e} / C_{s}$ is higher then more energy reservoir is available for the growth rate of the wave. Thats why the growth rate of the wave is larger for higher value of drift velocity and smaller for the lower drift velocity of the electrons. The same trend of growth rate is observed as discussed for Fig. 1.

The three dimensional surface plot the growth rate $\gamma / \omega_{r}$ of the quaiselectrostatic DIA twisted wave is shown in Fig. 3 against the product of ratio of number densities of dust to ion species and dust charging parameter $\eta Z_{d}$ and azimuthal parameter $\Upsilon=k / l q \theta$. The figure clearly illustrates the decreasing trend of the growth rate $\gamma / \omega_{r}$ with increasing $\eta Z_{d}$, while increasing trend is observed if the $\Upsilon=k / l q \theta$ is increased. This is due to the fact that the increasing magnitude of $\eta Z_{d}$ refers to charging to dust particles via capturing of electron species. If the dust charge is higher (means concentration of drifted electrons captured by neutral dust

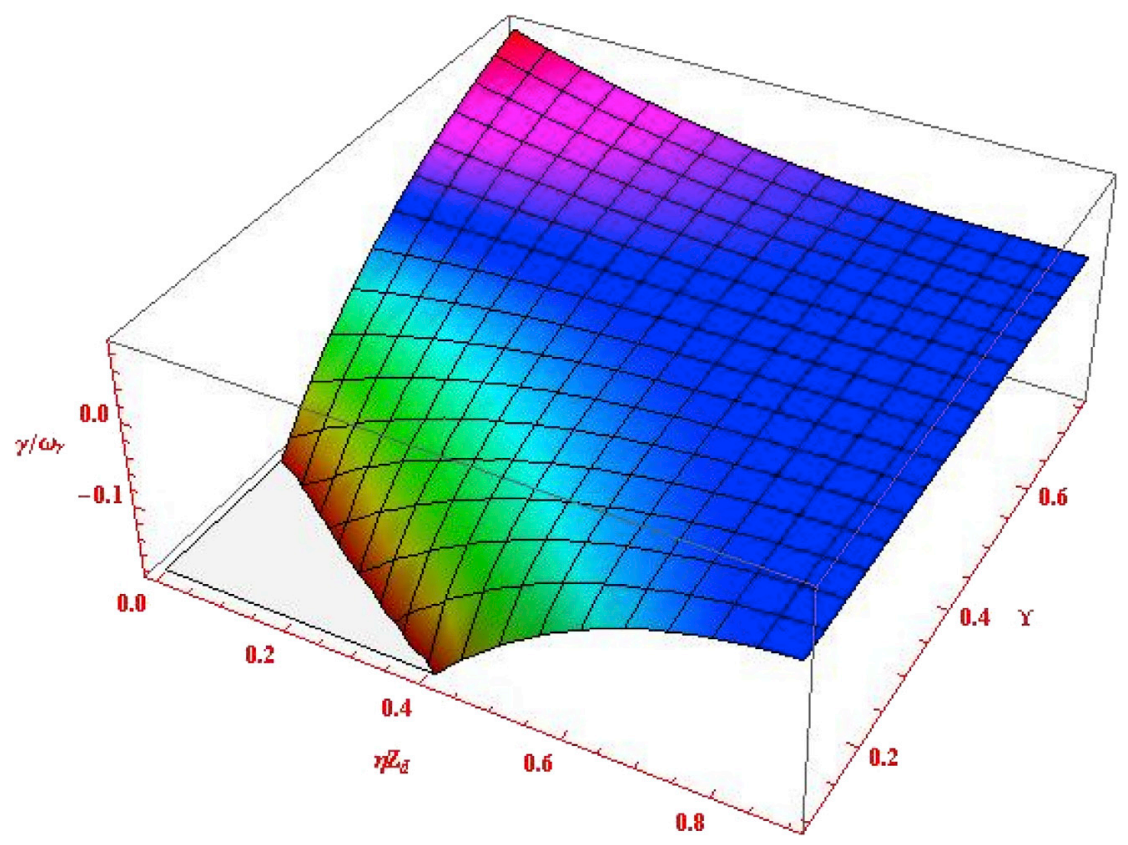

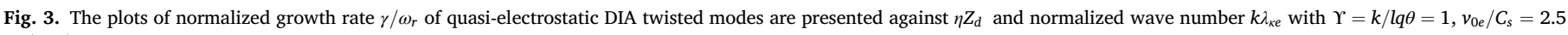
and $T_{e} / T_{i}=30$. 


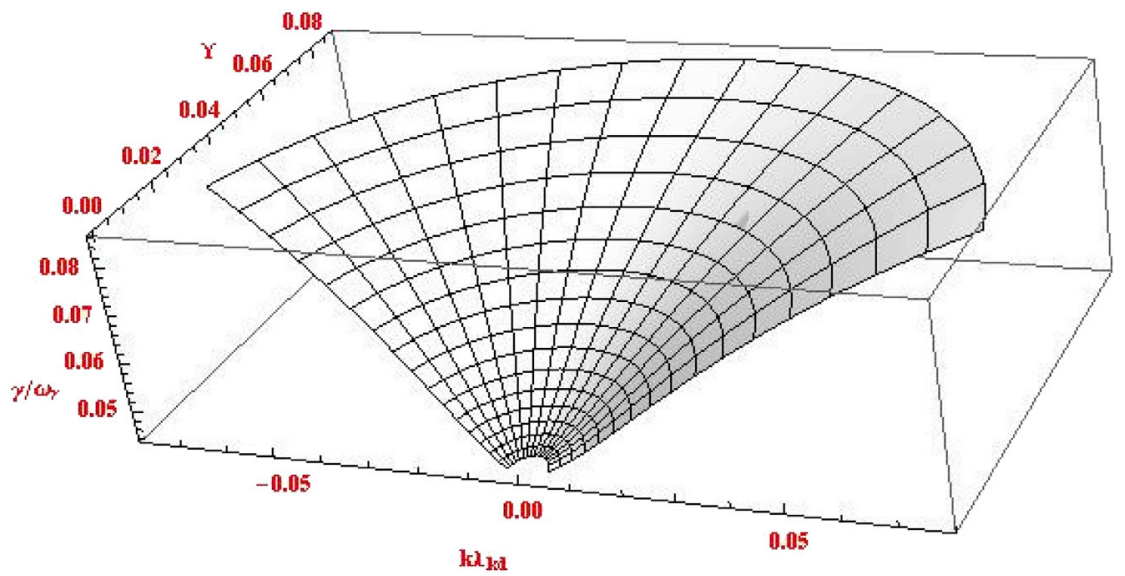

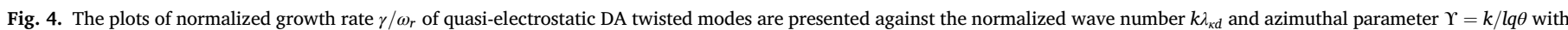
$\eta Z_{d}=0.01, v_{0 e} /\left(T_{i} / m_{d}\right)=2.5$ and $T_{e} / T_{i}=30$.

particles is higher) then the number of drifted electrons is lower to excite the wave. Therefore the growth rate of the quasi electrostatic DIA twisted mode is larger at smaller values of $\eta Z_{d}$ and vice versa. If we consider the effect of the azimuthal parameter $\Upsilon=k / l q \theta$ separately, the growth rate of quasi-electrostatic waves increases with increase in $\Upsilon=k / l q \theta$. Because the larger value of $\gamma=k / l q \theta$ reveals that the value of longitudinal wave vector $k$ is if larger as compare to $l q \theta$, then wave can grow to larger magnitude in comparison to the smaller value of longitudinal wave vector $k$.

In Fig. 4 describes the growth rate $\gamma / \omega_{r}$ of the quais-electrostatic DA twisted wave against the normalized wave number $k \lambda_{\kappa d}$ and azimuthal parameter $\mathrm{Y}=k / \mathrm{lq} \theta$. It is evident from the figure that with increasing values $k \lambda_{k d}$ and $\Upsilon=k / l q \theta$, the growth rate of the wave increases. The physical interpretation of this phenomenon, is the probability of more superthermal particles with increased normalized wave number $k \lambda_{\kappa d}$,

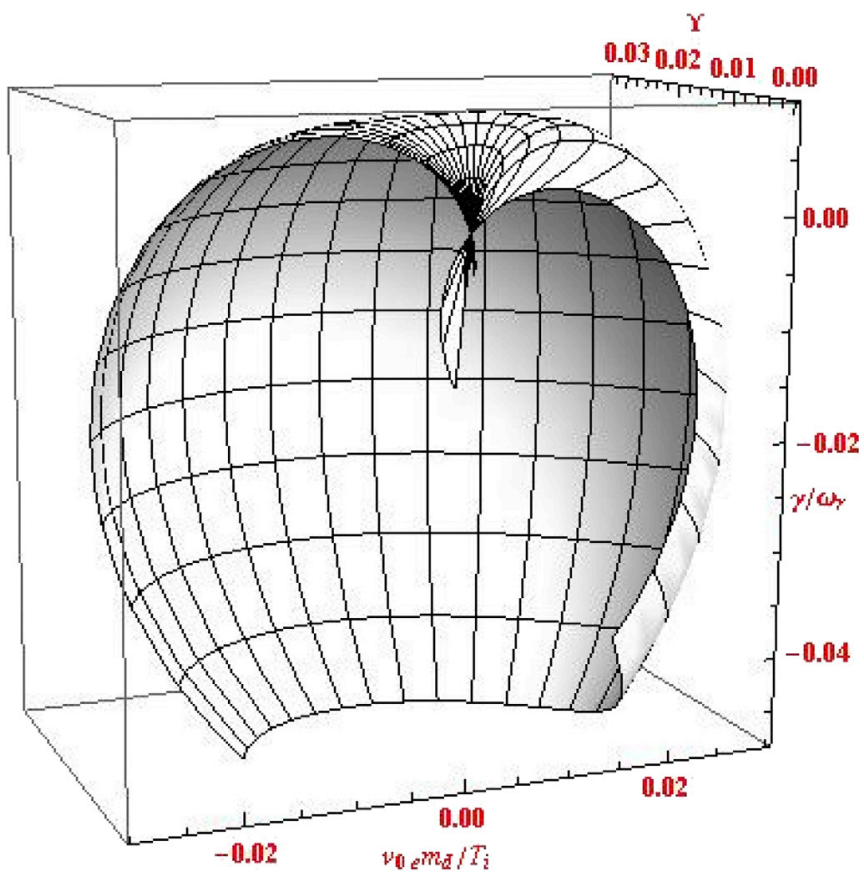

Fig. 5. The plots of normalized growth rate $\gamma / \omega_{r}$ of quasi-electrostatic DA twisted modes are presented against the normalized drift velocity $v_{0 e} /\left(T_{i} / m_{d}\right)$ and with azimuthal parameter $\Upsilon=k / l q \theta$ with normalized wave number $k \lambda_{x d}=0.1 \eta Z_{d}=0.01, v_{0 e} / C_{s}=2.5$ and $T_{e} / T_{i}=30$. available to excite the wave. This results in the increase of growth rate of quasi-electrostatic DA twisted wave with increasing $k \lambda_{k d}$. The increasing trend is observed in the growth rate $\gamma / \omega_{r}$ with the increasing value of azimuthal parameter $\Upsilon=k / l q \theta$. Because the larger value of $\Upsilon=k / l q \theta$ reveals that the value of longitudinal wave vector $k$ is if larger as compare to $l q \theta$, then wave can grow to larger magnitude in comparison to the smaller value of longitudinal wave vector $k$. The steepness of the curvature is more at smaller $\Upsilon$ because of larger $l q \theta$ and less at smaller $\Upsilon$ due to smaller $l q \theta$. It is depicted that the phasor wave number $l q \theta$ if dominates the spatial wave number $k$, then the wave has less chances to propagate in the longitudinal direction and results in the decreased value of the growth rate.

Fig. 5 presents the growth rate $\gamma / \omega_{r}$ of the quais-electrostatic DA twisted wave against the normalized drift velocity $v_{0 e} /\left(T_{i} / m_{d}\right)$ and azimuthal parameter $\Upsilon=k / l q \theta$. It is clear from the figure that by increasing the parameters $v_{0 e} /\left(T_{i} / m_{d}\right)$ and $\Upsilon=k / l q \theta$, the growth rate of the wave rate of the wave increases. Because the drift velocity acts as an energy source for the growth rate of the wave. If the drift velocity $v_{0 e} /\left(T_{i} / m_{d}\right)$ is higher then more energy reservoir is available for the wave to take energy and for the low drift velocity then energy reservoir has less to be taken by the wave. The same increasing trend is observed in the growth rate $\gamma / \omega_{r}$ with the increasing value of azimuthal parameter $\Upsilon=k / l q \theta$. Because the higher value of $\Upsilon=k / l q \theta$ reveals that the value of longitudinal wave vector $k$ is if larger as compare to $l q \theta$, then wave can grow to larger magnitude in comparison to the smaller value of longitudinal wave vector $k$.

The three dimensional surface plot is shown for the growth rate $\gamma / \omega_{r}$ of the quasi-electrostatic DA twisted wave in Fig. 6 against the product of dust to ion number densities ratio and dust charging parameter $\eta Z_{d}$ and azimuthal parameter $\mathrm{r}=k / l q \theta$. The figure clearly illustrates the decreasing trend of the growth rate $\gamma / \omega_{r}$ with increasing $\eta Z_{d}$, while increasing trend is observed if the $r=k / l q \theta$ is increased. This is due to the fact that the increasing magnitude of $\eta Z_{d}$ refers to charging to dust particles via capturing of electron species. If the dust charge is higher (means concentration of drifted electrons captured by neutral dust particles is higher) then the number of drifted electrons is lower to excite the wave. Therefore the growth rate of the quasi electrostatic DA twisted mode is larger at smaller values of $\eta Z_{d}$ and vice versa. If we consider the effect of the azimuthal parameter $\Upsilon=k / l q \theta$ separately, the growth rate of quasi-electrostatic waves increases with increase in $\Upsilon=k / l q \theta$. Because the larger value of $\mathrm{Y}=k / \mathrm{lq} \theta$ reveals that the value of longitudinal wave vector $k$ is if larger as compare to $l q \theta$, then wave can grow to larger magnitude in comparison to the smaller value of longitudinal wave vector $k$. 


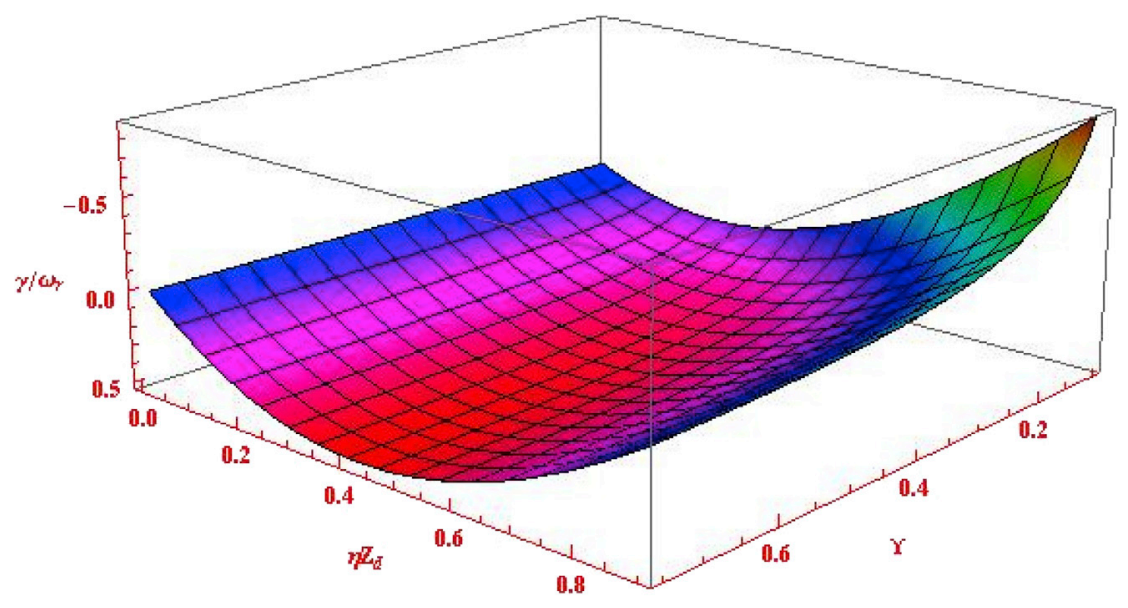

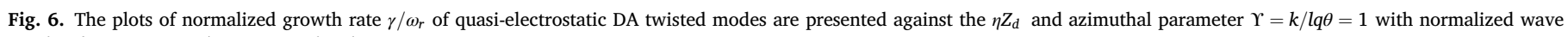
number $k \lambda_{\kappa d}=0.1, v_{0 e} / C_{s}=2.5$ and $T_{e} / T_{i}=30$.

\section{Discussion and conclusions}

The quasi-electrostatic DIA and DA twisted modes are investigated in the presence of helical electric field carrying orbital angular momentum. By applying Laguerre Gaussian mode function, the modes are decomposed into planar and non-planar components refer to spatial and phasor wave numbers. The behavior of the growth rate is observed with various vital parameters.

In dusty plasmas the superthermal population of particles is ubiquitous. From the composite spectra of CAPS/ELS $(0.6 \mathrm{eV}-26 \mathrm{keV})$ and the MIMI/LEMMS (15 keV-10 MeV) instruments on board Cassini (Schippers et al., 2008), the redial populations of the electron in Saturn's magnetosphere is observed. The thermal electrons depleted beyond 15 Rs while superthermal electrons still appeared in the middle and outer magnetosphere. The calibrated measurements of electrons between $10 \mathrm{eV}$ and $5.95 \mathrm{keV}$ by the Voyager Plasma Science Experiment (PLS) (Scudder et al., 1981) indicates that the electron distribution functions within the plasma sheet to be non-Maxwellian in the Jupiter's bow shock. Gaelzer et al., 2010 illustrates realistic description (solar wind electrons are described by an isotropic ${ }^{\circ}$ distribution and the protons are described by a Maxwellian) of the energization of particles and the turbulent cascade of magnetic fluctuations in solar and stellar wind. The velocity space distribution of the electrons and protons in space plasmas e.g., extended corona, solar wind and planetary magnetospheres, are well defined by power laws in particle speed such as Kappa distribution (Pierrard and Lazar, 2010; Lazar et al., 2012), most probably due to their acceleration mechanism in the presence of broadband lower hybrid or Alfven wave turbulence (Leubner, 2000). The dust-ion-acoustic (DIA) double layers (Mamun and Islam, 2011) in non-thermal dusty plasma (containing non-thermal electrons, inertial ions, and stationary positively as well as negatively charged dust) has been studied by deriving a modified Gardner (MG) equation to identify the non-planar (cylindrical and spherical) DIA double layers. Hellberg et al., 2005 reveals the possibility of Kappa-Maxwellian distribution in the space environments. Moreover, the instability described in the presented paper can explain the origin of waves and the enhance fluctuations (Verheest, 1996) observed in dusty plasmas like planetary magnetospheres (Arshad et al., 2014a,b,c), cometary tails (Arshad and Mirza, 2014) or even the solar wind (Arshad et al., 2014a,b,c) after such an event. It also describes the important role of waves and fluctuations in the transport processes in space plasmas (Gombosi, 2004) where the role of binary collisions (Tigik et al., 2016) is taken by the wave-particle interactions (Skiff et al., 2000) For very small drift velocity and large spectral index such that drift velocity approaches to zero and spectral index approaches to infinity, our results approaches to that presented in Ref. (Ali et al., 2016). But if we ignore the orbital angular momentum of the electric field and consider the planar electric field, then with large Kappa and zero drift velocity of electron, the results of Ref. (Baluku and Hellberg, 2015) can be obtained.

\section{Acknowledgment}

KA and ML acknowledges 8th International conference on the Physics of Dusty Plasmas (8ICPDP) to support the publication of the present studies under prestigious conference proceedings. These results were obtained in the framework of the projects G0A2316N (FWO-Vlaanderen), GOA/2015-014 (KU Leuven), C 90347 (ESA Prodex), and SCHL 201/35-1 (DFG-German Research Foundation).

\section{References}

Arshad, K., Rehman, A., Mahmood, S., 2016. Phys. Plasmas 23, 052107.

Akram, M.R., Gui, L., Liu, D., 2016. 7th Asia Pacific International Symposium on Electromagnetic Compatibility.

Alexandrov, A.F., Bogdankevich, L.S., Rukhadze, A.A., 1984. Principles of Plasma Electrodynamics. Springer-Verlag, Berlin, Heidelberg, New York, Tokio.

Ali, S., Bukhari, S., Mendonca, J.T., 2016. Phys. Plasmas 23, 033703.

Allen, L., Padgett, M., 2007. J. Mod. Opt. 54, 487.

Arshad, K., Mahmood, S., 2010. Phys. Plasmas 17, 124501.

Arshad, K., Mirza, A.M., 2014. Astrophys. Space Sci. 349, 753.

Arshad, K., Mahmood, S., Mirza, A.M., 2011. Phys. Plasmas 18, 092115.

Arshad, K., Siddique, F., Mirza, A.M., Rehman, A., 2014a. Astrophys. Space Sci. 350, 169.

Arshad, K., Ehsan, Z., Khan, S.A., Mahmood, S., 2014b. Phys. Plasmas 23, 023704.

Arshad, K., Mirza, A.M., Rehman, A., 2014c. Astrophys. Space Sci. 350, 585.

Arshad, K., Rehman, A., Mahmood, S., 2015. Phys. Plasmas 22, 112114.

Arshad, K., Lazar, M., Mahmood, S., Rehman, A., Poedts, S., 2017. Phys. Plasmas 24, 033701.

Baluku, T.K., Hellberg, M.A., 2015. Phys. Plasmas 22, 083701.

Barkan, A., D'Angelo, N., Merlino, R.L., 1996. Planetray Space Sci. 44, 239.

Barnett, S.M., Allen, L., Cameron, R.P., Gilson, C.R., Padgett, M.J., Speirits, F.C., Yao, A.M., 2016. J. Opt. 18, 064004.

Bessaa, A., Djebli, M., 2016. Phys. Plasmas 23, 113703.

Bialynicki-Birula, I., Bialynicka-Birula, Z., 2016. New J. Phys. 18, 023022.

Bret, A., 2009. Astrophys. J. 699, 990.

Davidson, R.C., 2014. Plasma Fusion Center. Massachusetts Institute of Technology, Cambridge, Massachusetts 02139, USA.

Fouial, N., Tahraoui, A., Annou, R., 2016. Phys. Plasmas 23, 113702.

Frank Verheest, F., 1992. Planet. Space Sci. 40, 1.

Gaelzer, R., Juli, M. C. de, Ziebell, L.F., 2010. J. Geophys. Res. 115, A09109.

Gao, Yi-Tian, Tian, Bo, 2006. Phys. Plasmas 13, 112901.

Gao, Yi-Tian, Tian, Bo, 2007. EPL 77.

Gombosi, T.I., 2004. Physics of the Space Environment. Cambridge University Press. Grier, D., 2003. Nature 424, 810.

Hamilton, D.P., Kruger, H., 2008. Nature 453, 72.

Hellberg, M.A., Mace, Richard, Cattaert, Tom, 2005. Space Sci. Rev. 121, 127.

Jack, B., Leach, J., Romero, J., Padgett, M.J., 2009. Phys. Rev. Lett. 103, 083602.

Jesacher, A., Furhapter, S., Bernet, S., Ritsch-Marte, M., 2005. Phys. Rev. Lett. 94, 233902.

Jukui, Xue, He, Lang, 2003. Phys. Plasmas 10, 339.

Khalil, S.M., Albaltan, W.S., 2015. Int. J. A A 5, 248. 
Khotyaintsev, M., 2006. Radar Probing of the Sun. Ph.D. thesis. Uppsala University, Uppsala, Sweden.

Khotyaintsev, M.V., Melnik, V.N., Thide, B., Konovalenko, O.O., 2006. Combination scattering by anisotropic Langmuir turbulence with application to solar radar experiments. Sol. Phys. 234, 169.

Knappmiller, S., Rapp, M., Robertson, S., Gumbel, J., 2011. J. Atmos. Sol. Terr. Phys. 73 2212.

Lazar, M., Schlickeiser, R., Poedts, S., Tautz, R.C., 2008. Mon. Not. R. Astron. Soc. 390 168.

Lazar, M., Schlickeiser, R., Poedts, S., 2012. Exploring the Solar Wind (INTECH), ISBN 978-953-51-0339-4.

Leubner, M.P., 2000. Planet. Space Sci. 48, 133.

Leyser, T.B., Norin, L., McCarrick, M., Pedersen, T.R., Gustavsson, B., 2009. Phys. Rev. Lett. 102, 065004.

Mamun, A.A., Islam, S., 2011. J. Geophys. Res. 116, A12323.

Mamun, A.A., Cairns, R.A., Shukla, P.K., 1996. Phys. Plasmas 3, 2610.

Mann, I., Pellinen-Wannberg, A., Murad, E., Nemecek, Z., 2011. Space Sci. Rev. 161, 1.

Mendonca, J.T., Thide, B., Then, H., 2009. Phys. Rev. Lett. 102, 185005.

Molina-Terriza, G., Torres, J.P., Torner, L., 2007. Nat. Phys, 3, 305.

Novo, A.S., Yoon, P.H., Lazar, M., Schlickeiser, R., Poedts, S., Seough, J., 2015. Phys. Plasmas 22, 092301

Oemrawsingh, S., Ma, X., Voigt, D., Aiello, A., Eliel, E., Hooft, G. t., Woerdman, J.P., 2005 Phys. Rev. Lett. 95, 240501.

Padgett, M., Bowman, R., 2011. Nat. Phot. 5, 343.

Patzold, M., Andert, T., Hahn, M., Asmar, S.W., Barriot, J.P., Bird, M.K., Hausler, B., Peter, K., Tellmann, S., Grun, E., Weissman, P.R., Sierks, H., Jorda, L., Gaskell, R., Preusker, F., Scholten, F., 2008. Nature 530, 63.

Pieper, J.B., Goree, J., 1996. Phys. Rev. Lett. 77, 3137.

Pierrard, V., Lazar, M., 2010. Sol. Phys. 267, 153.

Popovich, P., Umansky, M.V., Carter, T.A., Friedman, B., 2010. Phys. Plasmas 17, 1223125.
Rodrigues, J.D., Tercas, H., Mendonca, J.T., 2016. Europhys. Lett. 113, 13001.

Schippers, P., Blanc, M., AndrÃ $\odot$, N., Dandouras, I., Lewis, G.R., Gilbert, L.K.,

Persoon, A.M., Krupp, N., Gurnett, D.A., Coates, A.J., Krimigis, S.M., Young, D.T., Dougherty, M.K., 2008. J. Geophys. Res. 113, A07208.

Scudder, J.D., Sittler Jr., E.C., Bridge, H.S., 1981. J. Geophys. Res. A10, 8157.

Shukla, P.K., 2011. Phys. Plasmas 19, 083704.

Shukla, P.K., 2012. Phys. Lett. A 376, 2792.

Shukla, P.K., 2013. J. Geophys. Res. Space Phy. 118, 1-4.

Shukla, P.K., Eliasson, B., Stenflo, L., 2012. Phys. Rev. E 86, 016403.

Skiff, F., Ng, C.S., Bhattacharjee, A., Noonan, W.A., Case, A., 2000. Plasma Phys. control. Fusion 42, B27-B35.

Tamburini, F., Thide, B., Molina-Terriza, G., Anzolin, G., 2011. Nat. Phys. 7, 195.

Tian, Bo, Gao, Yi-Tian, 2005. Phys. Lett. A 340, 343.

Tigik, S.F., Ziebell, L.F., Yoon, P.H., Kontar, E.P., 2016. A\&A 586, A19.

Torney, M., Mormolino, C., Angelis, U. De, 2006. J. Plasma Phys. 72, 189.

Verbeeck, H.T.J., Schattschneider, P., 2010. Nature 467, 301.

Verheest, F., 1996. Space Sci. Rev. 77, 267.

Vieira, J., Trines, R.M.G.M., Alves, E.P., Fonseca, R.A., Mendonc, J.T., Bingham, R., Norreys, P., Silva, L.O., 2016. Nat. Commun. 7, 10371.

Vladimirov, S.S., Ostrikov, K., Sarnarian, A.A., 2005. Physics and Applications of Complex Plasmas. Imperial college press and references therein.

Wang, J., Yang, J., Fazal, I.M., Ahmed, N., Yan, Y., Huang, H., Ren, Y., Yue, Y., Dolinar, S., Tu, M., Willner, A.E., 2012. Nat. Phot. 6, 488.

Wedemeyer, S., Scullion, E., Steiner, O., de la Cruz Rodriguez, J., van der Voort, L.R., 2013. J. Phys. Conf. Ser. 440, 012005.

Youdin, A.N., Goodman, J., 2005. Astrophys. J. 620, 459.

Zhang, H., Zhang, W., Xi, L., Tang, X., Zhang, X., Zhang, X., 2016. IEEE Phot. Tech. Lett. 28, 1436.

Zolesi, B., Cander, L.R., 2014. Ionospheric Prediction and Forecasting. Springer Geophysics. 